\title{
Feasibility, safety, and indications for pure laparoscopic donor right posterior sectionectomy based on surgical techniques and outcomes of donors and recipients after living donor liver transplantation
}

\author{
Chan Woo Cho ${ }^{1}$, Gyu-Seong $\mathrm{Choi}^{2}$, Kyeong Sik Kim ${ }^{3}$ \\ ${ }^{1}$ Division of Transplantation, Department of Surgery, Yeungnam University Medical Center, Daegu, Korea \\ ${ }^{2}$ Division of Transplant Surgery, Department of Surgery, Samsung Medical Center, Seoul, Korea \\ ${ }^{3}$ Division of Transplantation, Department of Surgery, Soonchunhyang University Seoul Hospital, Seoul, Korea
}

Background: Right posterior section (RPS) graft for living donor liver transplantation (LDLT) is an alternative graft in a live liver donor with insufficient remnant left lobe volume and portal vein (PV) anomaly. However, there have been no reports regarding laparoscopic donor hepatectomy (LDH) for RPS graft. Herein, we report the results of pure laparoscopic donor right posterior sectionectomy (PLDRPS) in living donors with PV anomalies and the postoperative outcomes of donors and recipients who underwent PLDRPS and LDLT, respectively.

Methods: Seven donors (19-45 years) with PV anomaly underwent PLDRPS for seven LDLT recipients between June 2019 and June 2020. We reviewed the indications of LDH for RPS grafts, reported our experience with PLDRPS, and evaluated postoperative outcomes of donors and recipients.

Results: Indications for RPS graft selection were as follows: estimated left hemiliver volume less than $30 \%$ of whole liver volume (WLV); early branching right posterior PV such as type 2 or type 3 PV; estimated mismatch area volume less than $10 \%$ of WLV; right posterior hepatic duct running through the ventral side of the right PV; and RPS graft volume more than $40 \%$ of the recipients' standard liver volume. There was no open conversion or perioperative blood transfusion in donors. The operation time ranged from $219 \mathrm{~min}$ to $405 \mathrm{~min}$. Donors were discharged on postoperative days 6 to 16 without major complications. Out of seven recipients, three experienced major complications such as hepatic artery thrombosis, PV thrombosis, and bile leakage. One of these patients died of sepsis 6 months after LDLT.

Conclusions: PLDRPS in liver donors with PV anomaly was technically feasible and safe with experienced surgeons. Further evaluation of recipient outcomes in a larger number of cases is necessary to determine the usefulness of PLDRPS.

Corresponding author: Gyu-Seong Choi

E-mail: gyuseong.choi@samsung.com

() The Korean Society for Transplantation

This is an Open Access article distributed under the terms of the Creative Commons Attribution Non-Commercial License (http://creativecommons.org/licenses/by-nc/4.0/) which permits unrestricted non-commercial use, distribution, and reproduction in any medium, provided the original work is properly cited. 